\title{
Determination of functional properties of cocoa waste from concentrated cocoa drink
}

\begin{abstract}
Recovery of phenolic content from waste is one of the main concerns for any possible applications. This study was done to investigate and explore the functional properties of cocoa waste $(\mathrm{CW})$ from espresso cocoa production. The total phenolic content (TPC), total flavonoid content (TFC), 2,2-diphenyl-1-picrylhydrazyl scavenging assay (DPPH), metal $(\mathrm{Fe} 2+)$ chelating activity was determined. Parameters include water holding capacity (WHC), oil holding capacity (OHC), swelling capacity (SWC), proximate compositions, total dietary fiber (TDF), insoluble dietary fiber (IDF) and soluble dietary fiber (SDF) were also determined. As a reference spent coffee ground (SCG) was also studied under all parameters. Two solvents, ethanol and water were used to extract the bioactive compounds from $\mathrm{CW}$. The ethanol-CW extract was found to contain significantly the highest (p\&l0.05) TPC and TFC with $52.3 \mathrm{mg}$ GAE$/ \mathrm{ml}$ sample and $84.36 \mathrm{mg}$ Quercetin/ml sample respectively. This was

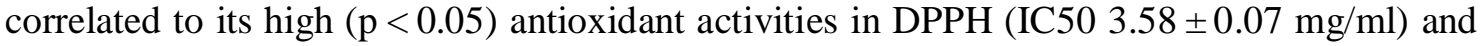

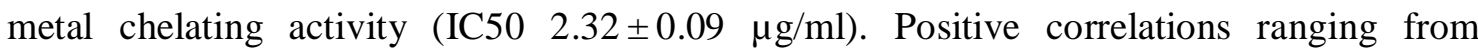
r2 1 国 82 to 0.98 were established between the phytochemicals and antioxidant activities of all extracts. All samples displayed significantly (pณl0.05) high WHC and SWC, in relevance to their high (p\&[0.05) TDF which were over $60 \%$ of $100 \mathrm{~g}$ dry matter. CW exhibited

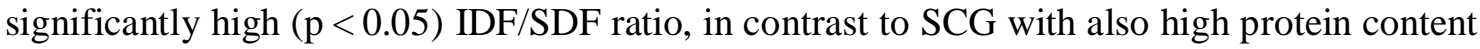
of $13 \%$. This study indicates that $\mathrm{CW}$ has a potential as a source of natural antioxidant and phytochemical in functional food development and intermediate food ingredient.
\end{abstract}

Keyword: Cocoa waste; Spent coffee grounds; Antioxidant; Ethanol extract; Water extract 